\title{
A novel quasi-oppositional modified Jaya algorithm for multi-objective optimal power flow solution
}

\begin{abstract}
This study introduces a novel meta-heuristic optimization algorithm known as quasioppositional modified Jaya (QOMJaya) to solve different multi-objective optimal power flow (MOOPF) problems. An intelligence strategy called quasi-oppositional based learning is incorporated into the proposed algorithm to enhance its convergence property, exploration capability, and solution optimality. Significant modifications to the basic Jaya algorithm are done to create a modified Jaya (MJaya) algorithm that can handle the MOOPF problem. A fuzzy decision-making strategy is proposed and incorporated into the Jaya algorithm as selection criteria for best and worst solutions. A new criterion for comparing updated and current candidate solutions is proposed. The concept of Pareto optimality is used to extract a set of non-dominated solutions. A crowding distance measure approach is utilized to maintain the diversity of Pareto optimality. In addition, a novel external elitist repository is developed to conserve discovered non-dominated solutions and to produce true and well-distributed Pareto optimal fronts. The proposed algorithm is scrutinized and validated using the modified IEEE 30-bus test system. Simulation results reveal the proposed algorithm's ability to produce real and well-distributed Pareto optimum fronts for all considered multi-objective optimization cases. Furthermore, the obtained results disclose the superiority of the proposed QOMJaya algorithm over both the proposed MJaya algorithm and several previous algorithms in terms of solution optimality and feasibility.
\end{abstract}

Keyword: Multi-objective optimal power flow; Jaya method; Meta-heuristic; Fuel cost; Real power loss; Voltage stability improvement 